\title{
A2255: The first detection of filamentary polarized emission in a radio halo
}

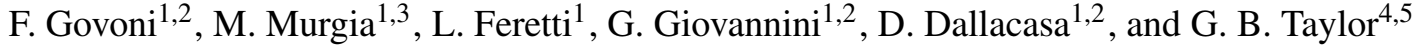 \\ ${ }^{1}$ Istituto di Radioastronomia - CNR/INAF, via Gobetti 101, 40129 Bologna, Italy \\ e-mail: fgovoni@ira.cnr.it \\ 2 Dipartimento di Astronomia, Univ. Bologna, Via Ranzani 1, 40127 Bologna, Italy \\ 3 INAF - Osservatorio Astronomico di Cagliari, Poggio dei Pini, Strada 54, 09012 Capoterra (CA), Italy \\ ${ }^{4}$ National Radio Astronomy Observatory, Socorro, NM 87801, USA \\ 5 Kavli Institute of Particle Astrophysics and Cosmology, Menlo Park, CA 94025, USA
}

Received 4 November 2004 / Accepted 26 November 2004

\begin{abstract}
A deep radio observation of the A2255 cluster of galaxies has been carried out at $1.4 \mathrm{GHz}$ with the Very Large Array synthesis telescope. Thanks to the excellent $(u, v)$ coverage and sensitivity achieved by our observation, the low brightness diffuse extended sources in the cluster (radio halo and relic) have been imaged with unprecedented resolution and dynamic range. We find that the radio halo has filamentary structures that are strongly polarized. The fractional linear polarization reaches levels of $\simeq 20-40 \%$ and the magnetic fields appear ordered on scales of $\sim 400 \mathrm{kpc}$. This is the first successful attempt to detect polarized emission from a radio halo and provides strong evidence that in this cluster the magnetic field is ordered on large scales.
\end{abstract}

Key words. galaxies: clusters : general - galaxies: intergalactic medium - polarization - magnetic fields radio continuum: general

\section{Introduction}

An ever increasing number of galaxy clusters exhibit largescale, diffuse, steep-spectrum synchrotron sources associated with the intracluster medium. These radio sources have been generally classified as radio halos or relics depending on their morphology and location. Radio halos are located at the cluster center and are characterized by a regular shape and extremely low surface brightness. Relics are similar, but are found at the cluster periphery and in general have an elongated shape. In some clusters, both a central halo and a peripheral relic are present. While relics are usually strongly polarized, no significant polarization has been detected so far in radio halos. In the Coma cluster, the upper limit to the halo fractional polarization is $\sim 10 \%$ at $1400 \mathrm{MHz}$ (Feretti \& Giovannini 1998). Good upper limits $(<5 \%)$ have been placed for the powerful radio halos of the galaxy clusters A2219, A2163 and 1E0657 - 57 (Bacchi et al. 2003; Feretti et al. 2001; Liang et al. 2000).

In addition to the analysis of the wide, diffuse synchrotron sources, cluster magnetic fields can be constrained through the detection of non-thermal emission of inverse Compton origin in the hard X-ray wavelengths or by studying Faraday rotation measure (RM) of polarized radio galaxies. It is known that these different methods of analysis give somewhat discrepant results for the magnetic field strength (see e.g. reviews by Carilli \& Taylor 2002; Govoni \& Feretti 2004, and references therein). The knowledge of the magnetic field structure may be the key issue to understand the origin of this discrepancy. Recently, Enßlin \& Vogt (2003), Vogt \& Enßlin (2003), Murgia et al. (2004), showed that the RM of radio galaxies can be used to infer not only the cluster magnetic field strength, but also the power spectrum of the cluster magnetic field fluctuations. Murgia et al. (2004) derived the magnetic field power spectrum of a sample of clusters for which good RM data of cluster radio galaxies were available. They found that A2255 appears as one of the clusters with a very steep intracluster magnetic field power spectra. Moreover they pointed out that morphology and polarization information of radio halos may provide important constraints on the power spectrum of the magnetic field fluctuations on large scales. In particular, their simulations showed that if the intracluster magnetic field fluctuates up to scales of some hundred $\mathrm{kpc}$, then steep magnetic field power spectra may give rise to detectable polarized filaments.

A2255 is a nearby $(z=0.0806$, Struble $\&$ Rood 1999), rich cluster which shows signs of undergoing a merger event. It is characterized by the presence of a diffuse radio halo source at the cluster center, a relic source at the cluster periphery, and several embedded head-tail radio galaxies (Jaffe \& Rudnick 1979; Harris et al. 1980; Burns et al. 1995; Feretti et al. 1997).

We observed A2255 with the purposes of detecting polarized emission from the radio halo and obtaining information on 


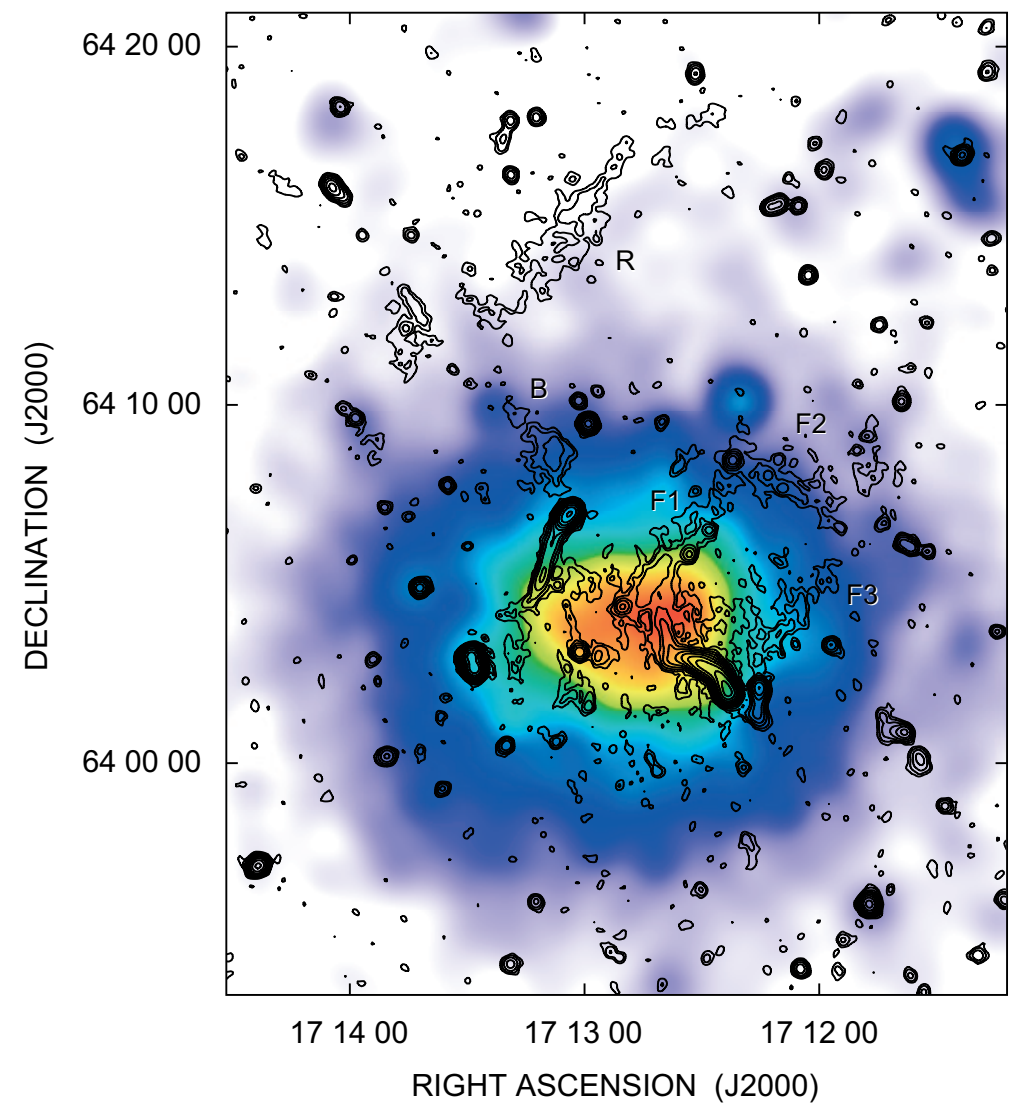

Fig. 1. Radio contours of the cluster A2255 overlaid on the Rosat X-ray image (colors). The radio image is at $1.4 \mathrm{GHz}$ and has a $F W H M$ of $15^{\prime \prime} \times 15^{\prime \prime}$ (uniform weighting). The sensitivity $(1 \sigma)$ is $16 \mu \mathrm{Jy} /$ beam and the dynamic range is $\simeq 6300$. Contour levels are: $0.05,0.1,0.2,0.4$, $0.8,1.6,3.2,6.4,12.8,25.6,51.2 \mathrm{mJy} /$ beam. No primary beam correction has been applied to the image.

the degree of ordering of the cluster magnetic field. In this letter we report the results of this high resolution and sensitivity observation.

Throughout this paper we assume a $\Lambda$ CDM cosmology with $H_{0}=71 \mathrm{~km} \mathrm{~s}^{-1} \mathrm{Mpc}^{-1}, \Omega_{\mathrm{m}}=0.3$, and $\Omega_{\Lambda}=0.7$. At the distance of A2255, $1^{\prime \prime}$ corresponds to $1.5 \mathrm{kpc}$.

\section{Radio data}

We observed A2255 with the Very Large Array (VLA) in C configuration on 2004 April 19, for a total integration time of $\simeq 11 \mathrm{~h}$ on source. This full-synthesis produced excellent $(u, v)$ coverage. A bandwidth of $25 \mathrm{MHz}$ was used for each of the two IF channels centered at $1465 \mathrm{MHz}$ and $1415 \mathrm{MHz}$. The observing frequencies were selected in order to avoid interferences. Faraday rotation effects between the two channels should be small for $R M \mathrm{~s}<100 \mathrm{rad} \mathrm{m}^{-2}$. The choice of the bandwidth was selected to reduce bandwidth smearing effects. Calibration and imaging were performed with the $\mathrm{NRAO}^{1}$ Astronomical Image Processing System (AIPS), following the standard procedure: Fourier-Transform, Clean and Restore. Self-calibration was applied to remove residual phase variations. Images in the Stokes parameters $I, Q$ and $U$ were made, using uniform and

1 The National Radio Astronomy Observatory (NRAO) is a facility of the National Science Foundation, operated under cooperative agreement by Associated Universities, Inc. natural weighting. The images of the polarized intensity (corrected for the positive bias), the fractional polarization and the position angle of polarization were derived from the $I, Q$ and $U$ images. The final images, shown here, were convolved with a circular Gaussian with a FWHM of $15^{\prime \prime}$ and $25^{\prime \prime}$ respectively.

\section{Results}

A full resolution $\left(F W H M=15^{\prime \prime}\right)$ image of A2255, including several radio galaxies, the central halo and the peripheral relic, is shown in Fig. 1 (contours). The radio emission is overlaid on the Rosat X-ray image (Feretti et al. 1997) to show the gas distribution of the cluster. The diffuse extended radio sources are resolved and at this high resolution only their brighter regions are visible. The relic (label R) appears as a long, straight filament of about 12' in length located $10^{\prime}$ to the North-East from the cluster center and elongated in the South-East NorthWest direction. The halo shows a complex morphology. The most prominent features detected at this resolution are straight filaments (labels F1, F2, F3), each about $6^{\prime}$ in length and $2^{\prime}$ in width, nearly perpendicular to each other. A bridge of low brightness emission (label B), seems to connect the halo and the relic on the northeast side. The filaments $\mathrm{F} 1$ and $\mathrm{F} 3$ are almost parallel to the relic, while the bridge $\mathrm{B}$ is nearly parallel to the filament F2.

In Fig. 2 (contours) a lower resolution $\left(F W H M=25^{\prime \prime}\right)$ image of the cluster radio emission is shown. Owing to the 

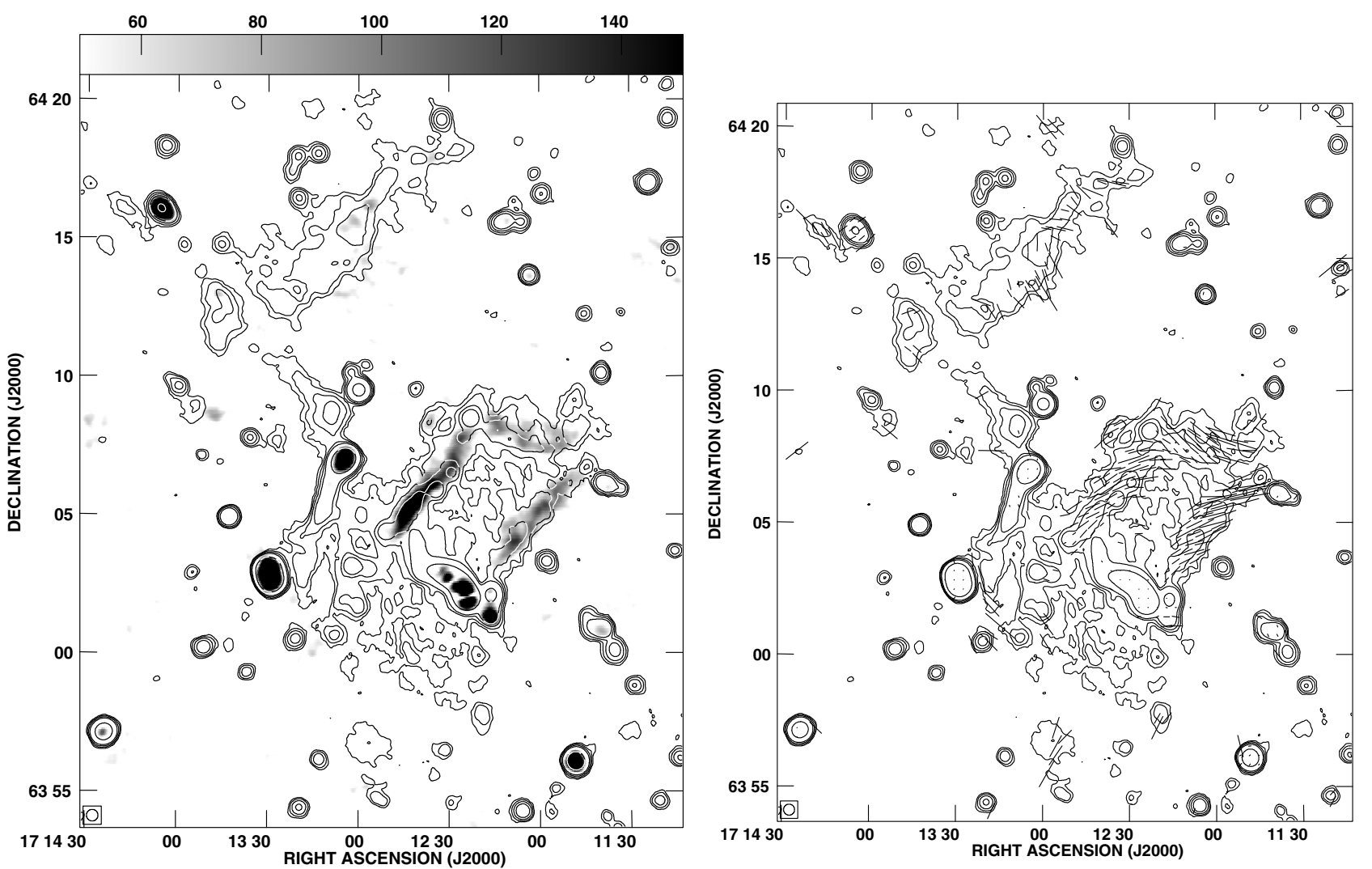

Fig. 2. Total intensity radio contours of A2255 at $1.4 \mathrm{GHz}$ with a $F W H M$ of $25^{\prime \prime} \times 25^{\prime \prime}$ (natural weighting). The sensitivity $(1 \sigma)$ is $\simeq 24 \mu \mathrm{Jy} /$ beam and the dynamic range is $\simeq 6000$. Contour levels are: $0.07,0.14,0.28,0.56,4.48 \mathrm{mJy} / \mathrm{beam}$. No primary beam correction has been applied. Left: the contours of the total intensity are overlaid on the linearly polarized intensity (grey-scale). The sensitivity ( $1 \sigma$ ) of the $U$ and $Q$ images is $\simeq 11 \mu \mathrm{Jy} /$ beam. The grey-scale shows the polarized flux from $50-150 \mu \mathrm{Jy} /$ beam. Right: the contours of the total intensity are overlaid on the polarization vectors. The vector orientation represents the projected E-field (not corrected for the contribution of the galactic rotation) while their length is proportional to the fractional polarization $\left(1^{\prime}=50 \%\right)$. All pixels with a fractional polarization less than $2 \sigma$, or with an error in the polarization angle greater than $10^{\circ}$, have been blanked.

higher signal to noise ratio, the low brightness regions of the diffuse sources are easily visible. On the left, the contours of the total intensity are overlaid on the linear polarized intensity (grey-scale). On the right, the contours of the total intensity are overlaid on the polarization vectors. The vector orientation shows the projected E-field and their length is proportional to the fractional polarization $\left(1^{\prime}=50 \%\right)$. In the figure, all pixels with a fractional polarization less than $2 \sigma$ or with an error in the polarization angle greater than $10^{\circ}$ were blanked. The polarized emission at $15^{\prime \prime}$ resolution (not shown here) displays similar results. The most important result is that the bright filaments of the halo appear strongly polarized at levels of $\simeq 20-40 \%$ ( $\simeq 4 \sigma-8 \sigma$ detections). Regions of ordered magnetic field of $\sim 400 \mathrm{kpc}$ in size can be observed. In the rest of the cluster we don't detect significant polarized emission except in the brighter regions of the relic where the fractional polarization is in the range $\simeq 15-30 \%(\simeq 3 \sigma-7 \sigma$ detections). The upper limit $(2 \sigma)$ to the fractional polarization in the fainter regions of the halo (i.e. where the average total intensity emission is about $0.15 \mathrm{mJy} /$ beam), is $\simeq 15 \%$. The galactic $\mathrm{RM}$ in the direction of $\mathrm{A} 2255$ is expected to be about $-6 \mathrm{rad} \mathrm{m}^{-2}$, based on the average of the RM galactic contribution published by Simard-Normandin et al. (1981) for sources near the cluster. Therefore, even if no Faraday rotation occurs within the cluster, the position angle of the E-field observed at $1.4 \mathrm{GHz}$ is rotated by $\sim 15^{\circ}$ clockwise with respect to the intrinsic (at $\lambda=0$ ) orientation. The electric polarization vectors of the relic tend to be roughly perpendicular to the relic elongation indicating aligned magnetic field structures within it, while the electric polarization vectors of the halo seems roughly parallel to the filaments.

Figure 3 shows the total intensity image at $25^{\prime \prime}$ resolution, with the discrete sources subtracted. The discrete sources were identified by making an image using long spacings, then their components were subtracted directly in the $u v$-plane (AIPS task UVSUB). To estimate the flux density of the cluster diffuse emission the primary beam correction was applied to the image in Fig. 3 (AIPS task PBCOR). The halo has a total flux density of $\simeq 56 \pm 3 \mathrm{mJy}$, the relic $\simeq 23 \pm 1 \mathrm{mJy}$, and their connecting bridge $\simeq 6 \pm 0.5 \mathrm{mJy}$. The three filaments F1, F2, F3 have flux densities of $9 \pm 0.5,3 \pm 0.5$, and $5 \pm 0.5 \mathrm{mJy}$ respectively, indicating a total flux $\simeq 30 \%$ of the flux of the entire halo.

\section{Discussion}

The absence of a significant polarization in halos has been interpreted as the result of two concurrent effects: internal Faraday rotation and beam depolarization. The thermal 
intracluster gas is mixed with the relativistic plasma, thus due to internal Faraday rotation, significant depolarization may occur within the radio halos. Moreover as a consequence of their extremely low surface brightness, radio halos have been studied so far at low spatial resolution. This could result in a significant decrease of the observed fractional polarization, if the cluster magnetic field is tangled on scales smaller than the beam.

Murgia et al. (2004) showed that if the outer scale of the magnetic field fluctuations extends up to some hundred $\mathrm{kpc}$, and if the power spectrum ${ }^{2}$ of the cluster magnetic field is relatively steep $(n \geq 3)$ there could be a chance of detecting filamentary polarized emission in the halo. The deep and high resolution radio observation of A2255 presented here confirms their prediction. The radio halo of A2255 shows, for the first time, filaments of strong polarized emission. Moreover, the distribution of the polarization angles indicates that the magnetic field of this cluster fluctuates up to scales of about $400 \mathrm{kpc}$ in size.

The detection of polarized emission in a synchrotron halo in A2255 opens up new questions regarding its nature, origin, and connection with the history of merging. The halo filaments could result from a compression wave, which enhances and aligns disordered magnetic fields. Most turbulence theories involve the processes by which the energy is injected into a medium at large spatial scales and than converted into motions at smaller and smaller spatial scales until reaching scales at which it is dissipated. What we have detected in A2255 may be the injection in the intracluster medium of energy on large scales, produced for example by a shock during a cluster merger. However both the radio morphology of the filaments nearly perpendicular to each other, and the electric polarization vectors running roughly parallel to the elongation of the radio halo filaments (indicating magnetic field structures perpendicular within them) are quite unusual and difficult to explain in this framework. Spatial spectral index information, in conjunction with high resolution cluster $\mathrm{X}$-ray and temperature images, will test electron re-acceleration models (e.g. turbulence, shock) responsible for the halo filaments formation and could help to determine whether these structures are produced by shock waves resulting from a cluster merger.

Another important issue is to understand why the halo filaments are so strongly polarized. They could be cluster structures in foreground in which the Faraday rotation is negligible. RM information, obtained at well separated frequencies, will be of great importance to evaluate this possibility. In this case they should have a low rotation measure.

Finally it is not clear whether these filamentary polarized structures, are typical features of clusters or if A2255 is a peculiar case. One can devise a scenario in which all clusters have magnetic fields fluctuating both on small (visible through RM of radio galaxies) and large scales (visible through radio halos). But only for those clusters for which the power spectrum of the magnetic field fluctuations is steep enough will these polarized filamentary structures be detectable. Future

\footnotetext{
${ }^{2}\left|B_{k}\right|^{2} \propto k^{-n}$ where $n$ is the index of the power spectrum of the magnetic field fluctuations. The power spectrum is expressed as a vectorial form in $k$-space.
}

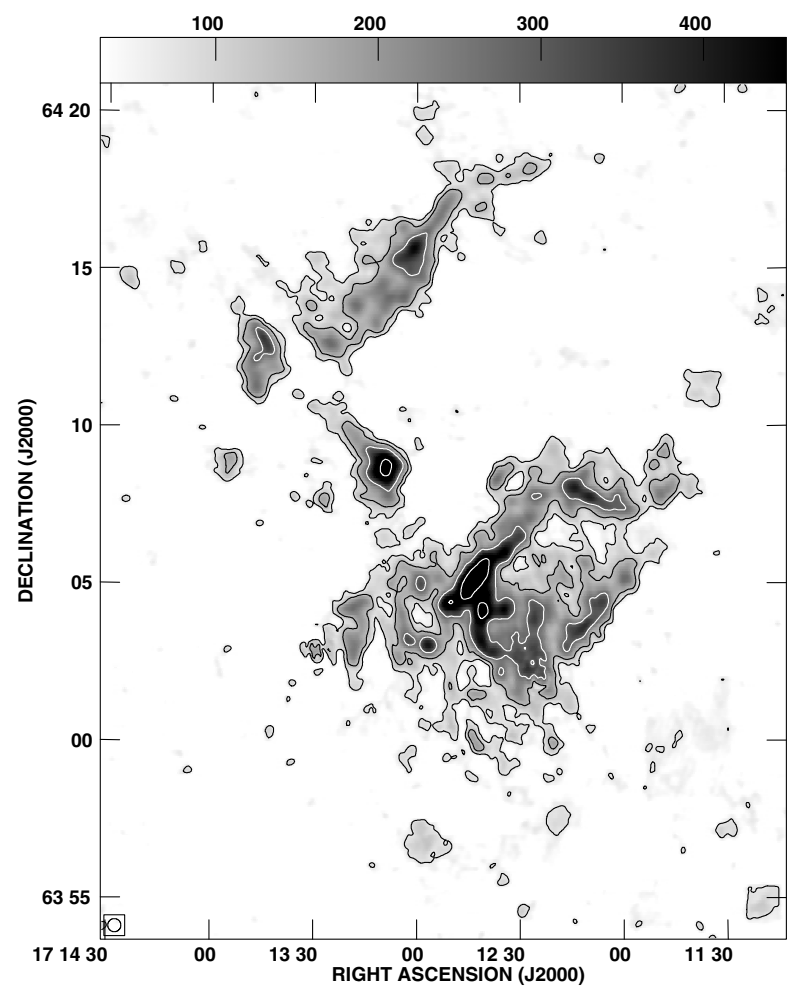

Fig. 3. The $1.4 \mathrm{GHz}$ image of the cluster radio emission after subtraction of the discrete sources. The $F W H M$ is $25^{\prime \prime} \times 25^{\prime \prime}$ (natural weighting). Contour levels are: $0.07,0.14,0.28,0.56 \mathrm{mJy} /$ beam. The grey scale flux is $30-450 \mu \mathrm{Jy} /$ beam. No primary beam correction has been applied to this image.

observations on other clusters containing radio halos, and selected on the basis of their magnetic field power spectrum, are necessary to test these ideas.

\section{References}

Carilli, C. L., \& Taylor, G. B. 2002, ARA\&A, 40, 319

Bacchi, M., Feretti, L., Giovannini, G., \& Govoni, F. 2003, A\&A, 400, 465

Burns, J. O., Roettiger, K., Pinkney, J., et al. 1995, ApJ, 446, 583

Enßlin, T. A., \& Vogt, C. 2003, A\&A, 401, 835

Feretti, L., Böhringer, H., Giovannini, G., \& Neumann, D. 1997, A\&A, 317, 432

Feretti, L., \& Giovannini, G. 1998, Untangling Coma Berenices: A New Vision of an Old Cluster, Proc. of the meeting held in Marseilles, France, ed. A. Mazure, F. Casoli, F. Durret, \& D. Gerbal (Word Scientific Publishing Co Pte Ltd), 123

Feretti, L., Fusco-Femiano, R., Giovannini, G., \& Govoni, F. 2001, A\&A, 373, 106

Govoni, F., \& Feretti, L. 2004, Int. J. Mod. Phys. D, 13, 1549

Harris, D. E., Kapahi, V. K., \& Ekers, R. D. 1980, A\&AS, 39, 215

Jaffe, W. J., \& Rudnick, L. 1979, ApJ, 233, 453

Liang, H., Hunstead, R. W., Birkinshaw, M., \& Andreani, P. 2000, ApJ, 544, 686

Murgia, M., Govoni, F., Feretti, L., et al. 2004, A\&A, 424, 429

Simard-Normandin, M., Kronberg, P. P., Button, S. 1981, ApJS, 45, $97 \mathrm{~S}$

Struble, M. F., \& Rood, H. J. 1999, ApJS, 125, 35

Vogt, C., \& Enßlin, T. A. 2003, A\&A, 412, 373 